\title{
Non-circular multi-core fibers for super-dense SDM
}

\author{
Md Nooruzzaman ${ }^{1 a)}$, Kunimasa Saitoh ${ }^{2}$, Yusuke Sasaki ${ }^{3}$, \\ Katsuhiro Takenaga ${ }^{3}$, Kazuhiko Aikawa ${ }^{3}$, and Toshio Morioka ${ }^{1}$ \\ ${ }^{1}$ Department of Photonics Engineering, Technical University of Denmark, \\ Kgs. Lyngby, 2800, Denmark \\ ${ }^{2}$ Graduate School of Information Science and Technology, Hokkaido University, \\ Sapporo 060-0814, Japan \\ ${ }^{3}$ Advanced Technology Laboratory, Fujikura Ltd., \\ 1440 Mutsuzaki, Sakura, Chiba 285-8550, Japan \\ a)noor_319@yahoo.com
}

Abstract: Non-circular multi-core fibers (NC-MCFs) to accommodate more than 100 cores per fiber are proposed for super-dense space-division multiplexing. The proposed MCFs have the potential for easier splicing/ connection and their applicability to highly-dense ribbon MCFs for ultrahigh capacity optical transport systems.

Keywords: Space-division multiplexing, non-circular multi-core fibers, ribbon multi-core fibers

Classification: Optical systems

\section{References}

[1] T. Morioka, "New generation optical infrastructure technologies: "EXAT Initiative" towards 2020 and beyond," OECC (2009) FT4 (invited) (DOI: 10. 1109/OECC.2009.5213198).

[2] R.-J. Essiambre, et al.: "Capacity limits of optical fiber networks," J. Lightwave Technol. 28 (2010) 662 (DOI: 10.1109/JLT.2009.2039464).

[3] D. J. Richardson, et al.: "Space-division multiplexing in optical fibres," Nat. Photonics 7 (2013) 354 (DOI: 10.1038/nphoton.2013.94).

[4] T. Morioka, et al:: "R\&D of $3 \mathrm{M}$ technologies towards the realization of exabit/s optical communications," IEICE Trans. Commun. E100B (2017) 1707 (DOI: 10.1587/transcom.2016PFI0018).

[5] Y. Amma, et al:: "High-density multicore fiber with heterogeneous core arrangement," OFC (2015) Th4C.4 (DOI: 10.1364/OFC.2015.Th4C.4).

[6] Y. Sasaki, et al.: "Crosstalk-managed heterogeneous single-mode 32-core fibre," European Conference on Optical Communication (2016) W.2.B.2.

[7] Y. Sasaki, et al.: "Single-mode 37-core fiber with a cladding diameter of $248 \mu \mathrm{m}$," OFC (2017) Th1H.2 (https://doi.org/10.1364/OFC.2017.Th1H.2).

[8] T. Mizuno, et al:: "32-core dense SDM unidirectional transmission of PDM16QAM signals over $1600 \mathrm{~km}$ using crosstalk-managed single-mode heterogeneous multicore transmission line," OFC (2016) PDP Th5C.3 (https://doi. org/10.1364/OFC.2016.Th5C.3).

[9] T. Kobayashi, et al.: "1-Pb/s (32 SDM/46 WDM/768 Gb/s) C-band dense SDM transmission over 205.6-km of single-mode heterogeneous multi-core fiber using 96-Gbaud PDM-16QAM channels," OFC (2017) PDP Th5B.1 (https://doi.org/10.1364/OFC.2017.Th5B.1). 
[10] T. Sakamoto, et al:: "Low-loss and low-DMD 6-Mode 19-core fiber with cladding diameter of less than $250 \mu \mathrm{m}$," J. Lightwave Technol. 35 (2017) 443 (DOI: 10.1109/JLT.2016.2610479).

[11] K. Saitoh, et al:: "Multicore fiber technology," J. Lightwave Technol. 34 (2016) 55 (DOI: 10.1109/JLT.2015.2466444).

[12] O. N. Egorova, et al.: "Multicore fiber with rectangular cross-section," Opt. Lett. 39 (2014) 2168 (DOI: 10.1364/OL.39.002168).

[13] G. A. Mahdiraji, et al.: "Multicore flat fiber: A new fabrication technique," IEEE Photonics Technol. Lett. 26 (2014) 1972 (DOI: 10.1109/LPT.2014. 2343637).

[14] T. Nagashima, et al.: "Multi-core fibre with concaved double-D shape cross section," Proc. ECOC2017 (2017) M.2.B.

[15] Y. Mitsunaga, et al.: "Failure prediction for long length optical fiber based on proof testing," J. Appl. Phys. 53 (1982) 4847 (DOI: 10.1063/1.331316).

[16] K. Nagano, et al.: "Change of the refractive index in an optical fiber due to external forces," Appl. Opt. 17 (1978) 2080 (DOI: 10.1364/AO.17.002080).

[17] R. Ulrich, et al:: "Bending-induced birefringence in single-mode fibers," Opt. Lett. 5 (1980) 273. (DOI: 10.1364/OL.5.000273).

\section{Introduction}

An exponential growth of global data traffic has been propelling demands for highcapacity optical transmission systems and networks. Optical fiber communication technologies have constantly made innovative inventions such as Erbium-doped fiber amplifiers (EDFAs) and digital coherent technology to substantially increase the system capacity based on single-mode fibers (SMFs) over the last three decades. The maximum capacity of the current SMF-based transmission systems, however, has been found to be up to around $100 \mathrm{Tbit} / \mathrm{s}$ per fiber due to nonlinear distortions of optical signals, limited bandwidths of optical amplifiers, and eventually the fiber fuse phenomenon $[1,2,3]$.

To overcome this looming capacity crunch, a new multiplexing technology, space-division multiplexing (SDM) has been explored over the last ten years [4]. In $\mathrm{SDM}$, the signal power is distributed over multiple spatial channels in the form of multiple modes and/or cores, thus lowering the power density, hence decreasing the nonlinear distortions and allowing for increased capacity and reach. Recently, single-mode (SM)-MCFs containing more than 30 cores have been fabricated and tested in high capacity transmission experiments $[5,6,7,8,9]$ and a few-mode (FM)-MCF containing 19 cores has been fabricated [10] with cladding diameters of less than $250 \mu \mathrm{m}$. However, because of a limited cladding diameter (CD) constraint of about $250 \mu \mathrm{m}$ to ensure comparable mechanical reliability to that of SMFs $[10,11]$, these number of cores are considered to be about the maximum ones that can be accommodated within a circular cladding cross section while keeping sufficient low inter-core crosstalk (XT) required for long-haul transmission systems.

In this paper, we report a conceptual design of non-circular multi-core fibers (NC-MCFs) having a non-circular cladding which have potentials of accommodating more than 100 cores for super-dense SDM while keeping comparable mechanical reliability to that of SMFs. The proposed NC-MCFs also have advantages of 
easier alignment for splicing and production of connectors as well as their applicability of forming super-dense ribbon fibers housing several hundred cores, which may be applicable for data-center interconnects, access, metro as well as long-haul systems.

\section{NC-MCF design model}

\subsection{Basic shapes and core layouts}

NC-MCFs have recently been proposed and fabricated [12, 13, 14]. Fig. 1 illustrates three examples of non-circular cross sections investigated in this article, namely, "elliptical", "racetrack oval shaped", and "sharp-cornered oblong shaped" (also referred to as "double D-shaped") claddings. The sharp-cornered oblong shaped MCFs can be formed, for example, by grinding two sides (top and bottom sides) of a circular preform while a D-shaped cross section can be formed by grinding one side. The number of cores in a NC-MCF depends on the types of cross sections as shown in Fig. 1. Assuming the same dimensions of $a$ and $b$ where $a$ is the cladding diameter in the major axis and $b$ in the minor axis, the elliptical MCF has the smallest area with a minimum numbers of cores, whereas the sharpcornered oblong shaped MCF has the largest area with the highest number of cores.
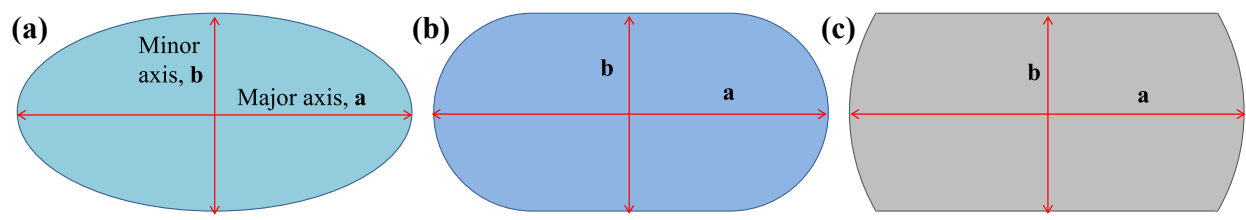

Fig. 1. Various cross-sections of non-circular MCFs with (a) elliptical (b) racetrack oval shaped, and (c) sharp-cornered oblong shaped claddings. The dimensions of the major and minor axes are "a" and "b", respectively.

Fig. 2 illustrates three major core arrangements for use in NC-MCFs. In Fig. 2(a), a hexagonal close packed structure (HCPS) layout is shown where cores are located at the corners of an equilateral triangle with a core pitch $\Lambda$ (a distance between any two neighboring cores) of $30 \mu \mathrm{m}$ as an example. Fig. 2(b) shows a square lattice structure (SLS) where cores are located at the corners of a square and in Fig. 2(c), a half-pitch shifted square lattice structure (HPS-SLS) is shown where cores in the two horizontal rows are shifted by half a pitch compared to SLS. In this structure, the horizontal core distance is $\Lambda_{a}$ (here $30 \mu \mathrm{m}$ ) while the distance between two vertical cores is $\Lambda_{b}=\Lambda_{a} \sqrt{5} / 2$ (here $33.5 \mu \mathrm{m}$ ). In HPS-SLS, although the number of cores which can be accommodated is almost the same as that of SLS, it has a clear advantage of having only two adjacent cores with a core distance $30 \mu \mathrm{m}$ in this example while the other four adjacent cores are farther with a core distance $33.5 \mu \mathrm{m}$, achieving less total inter-core XT.

\subsection{Fiber parameters and XT of NC-MCFs}

Average total XT is investigated for two/three types of trench-assisted heterogeneous core arrangements and a trench-assisted homogeneous core arrangement with the core parameters listed in Table I and illustrated in Fig. 3. All the cores are 

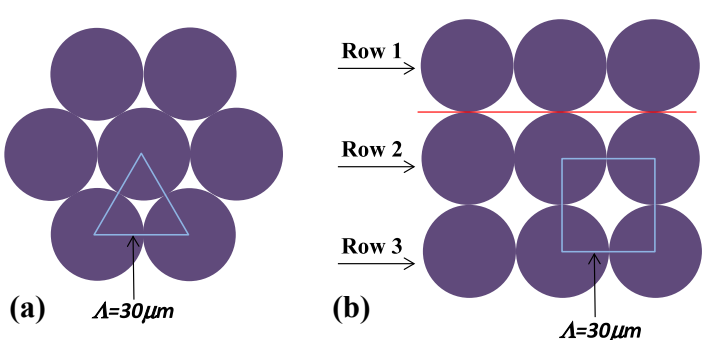

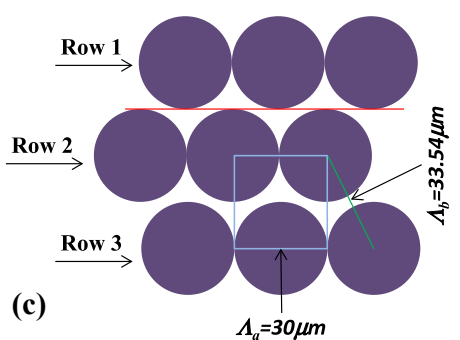

$\Lambda_{a}=30 \mu m$

Fig. 2. Examples of three major core arrangements of NC-MCFs. (a) Hexagonal close packed structure (HCPS), (b) square lattice structure (SLS), and (c) half-pitch shifted square lattice structure (HPS-SLS).

designed to have $A_{\text {eff }}$ of $80 \mu \mathrm{m}^{2}$ at $1550 \mathrm{~nm}$ with a cutoff wavelength of less than $1530 \mathrm{~nm}$ after $1 \mathrm{~km}$ propagation and a bending loss of $<0.1 \mathrm{~dB} / 100$ turns at a bending radius of $30 \mathrm{~mm}$ and at $1625 \mathrm{~nm}$. For the two/three types of heterogeneous cores, the effective refractive index difference $\Delta n_{\text {eff }}$ is $>0.00055$. The relative refractive index difference $\Delta_{t}$ between the trench and the cladding is assumed to be $-0.7 \%$. The inter-core XT between two cores with a core distance of $29 \mu \mathrm{m}$ was calculated for $100 \mathrm{~km}$ at $1550 \mathrm{~nm}$ as listed in Table II. For example, XT between the highest $(\mathrm{H})$ index and lowest $(\mathrm{L})$ index cores was found to be $-48.6 \mathrm{~dB}$ per $100 \mathrm{~km}$. The inter-core XT between two cores having the same refractive index (homogeneous core arrangement) was found to be $-20.7 \mathrm{~dB}$ per $100 \mathrm{~km}$. In this paper, the correlation length of the MCF was assumed to be $5 \mathrm{~m}[5,11]$. Different types of core arrangements are shown in Figs. 4 and 5.

Table I. Core parameters used in the proposed NC-MCFs.

\begin{tabular}{|c|c|c|c|}
\hline \multicolumn{4}{|c|}{ Three types of cores } \\
\hline & Core $\mathrm{H}$ & Core $\mathrm{M}$ & Core $\mathrm{L}$ \\
\hline$\Delta[\%]$ & 0.344 & 0.305 & 0.250 \\
\hline $\mathrm{r}_{1}$ & 4.78 & 4.61 & 6.01 \\
\hline $\mathrm{r}_{2} / \mathrm{r}_{1}$ & 1.7 & 1.7 & 1.0 \\
\hline $\mathrm{W} / \mathrm{r}_{1}$ & 0.70 & 0.79 & 0.92 \\
\hline
\end{tabular}

\begin{tabular}{|c|c|c|}
\hline \multicolumn{3}{|c|}{ Two types of cores } \\
\hline & Core a & Core $\mathrm{b}$ \\
\hline$\Delta[\%]$ & 0.35 & 0.31 \\
\hline $\mathrm{r}_{1}$ & 4.80 & 4.65 \\
\hline $\mathrm{r}_{2} / \mathrm{r}_{1}$ & 1.7 & 1.7 \\
\hline $\mathrm{W} / \mathrm{r}_{1}$ & 0.58 & 1.03 \\
\hline
\end{tabular}

\begin{tabular}{|c|c|}
\hline \multicolumn{2}{|c|}{$\begin{array}{c}\text { Homogeneous } \\
\text { core }\end{array}$} \\
\hline & Core a \\
\hline$\Delta[\%]$ & 0.35 \\
\hline $\mathrm{r}_{1}$ & 4.80 \\
\hline $\mathrm{r}_{2} / \mathrm{r}_{1}$ & 1.7 \\
\hline $\mathrm{W} / \mathrm{r}_{1}$ & 0.58 \\
\hline
\end{tabular}

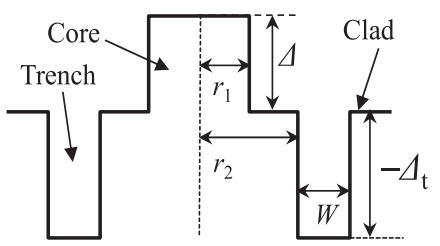

Fig. 3. Schematic of core profile.

Fig. 6 shows the calculated average total XT at $1550 \mathrm{~nm}$ for $100 \mathrm{~km} \mathrm{NC-MCFs.}$ The inter-core XT between two cores was calculated based on the coupled power theory [11]. Fig. 6(a) shows the XT values for heterogeneous NC-MCFs with three types of cores. HCPS has the highest XT as there are six surrounding cores with a distance of $\Lambda_{a}$. HPS-SLS has the lowest XT as only two cores are located at $\Lambda_{a}$ while the other four cores are at $\Lambda_{b}$ as explained in Fig. 2(c). 
Table II. Crosstalk between two cores of the proposed NC-MCFs.

\begin{tabular}{|c|c|c|c|}
\hline & \multicolumn{3}{|c|}{ Inter-core XT (in $\mathrm{dB}$ ) per $100 \mathrm{~km}$ for core to core distance $=29 \mu \mathrm{m}$} \\
\hline 3 types cores & Between H-M & Between M-L & Between H-L \\
\hline & -42.1 & -41.5 & -48.6 \\
\hline 2 types cores & Between a-b & Between a-a & Between b-b \\
\hline & -45.1 & -20.7 & -29.3 \\
\hline 1 type cores & \multicolumn{3}{|c}{-20.7} \\
\hline
\end{tabular}
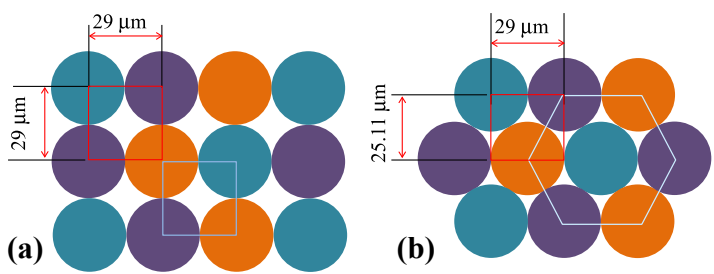

(c)

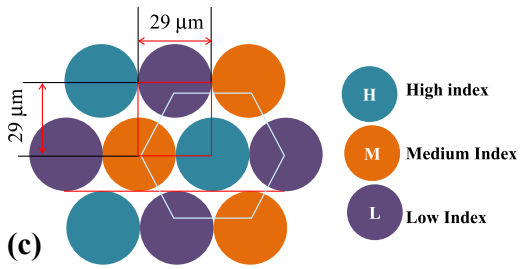

Fig. 4. Three types of core arrangements: (a) SLS, (b) HCPS and (c) HPS-SLS.
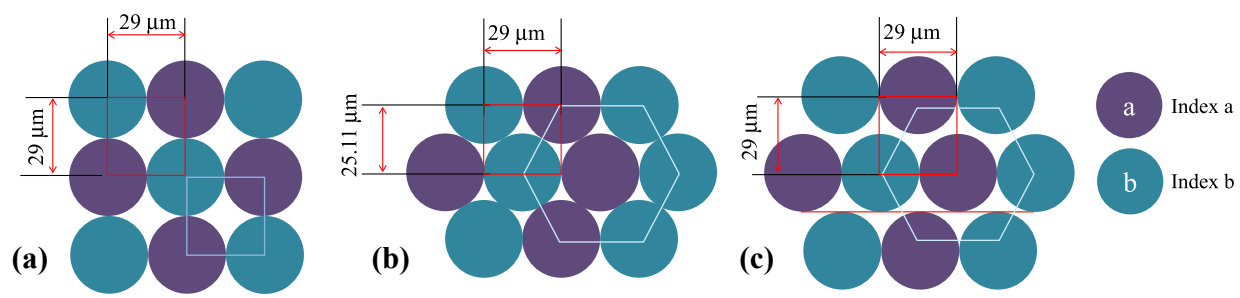

Fig. 5. Two types of core arrangements: (a) SLS, (b) HCPS and (c) HPS-SLS.

Fig. 6(b) shows the XT values for heterogeneous NC-MCFs with two types of cores. The total XT for HCPS and SLS is higher than those for three types of cores shown in Fig. 6(a). In HCPS, two same type of cores are located at a distance $\Lambda_{a}$, which contributed to the highest XT. In SLS, four same types of cores contributes to XT more although they are located at $\Lambda_{a} \sqrt{2}$ than the different index cores at $\Lambda_{a}$. On the other hand, for HPS-SLS, a single core is surrounded by two cores having a different refractive index at $\Lambda_{a}$, and the other four cores are at $\Lambda_{b}$, where two have the same refractive index. Finally, Fig. 6(c) shows the XT for homogeneous cores all having the same index profile.

It is noted from Fig. 6 that the heterogeneous NC-MCFs with three types of cores (HPS-SLS, $\Lambda_{a}=29 \mu \mathrm{m}$ ) have the lowest XT of $-39 \mathrm{~dB} / 100 \mathrm{~km}$ applicable for transmission distances up to $10,000 \mathrm{~km}$ with QPSK and 2,500 km with 16 QAM modulation formats, assuming the XT of $-19 \mathrm{~dB}$ and $-25 \mathrm{~dB}$ required for QPSK and 16 QAM transmission, respectively, under $0.5 \mathrm{~dB}$ penalty [8]. The heterogeneous NC-MCFs with two types of cores (HPS-SLS, $\Lambda_{a}=29 \mu \mathrm{m}$ ) on the other hand have XT of $-27 \mathrm{~dB} / 100 \mathrm{~km}$ applicable for transmission distances up to $600 \mathrm{~km}$ and $150 \mathrm{~km}$ with QPSK and 16 QAM modulation formats, respectively. The homogeneous NC-MCFs (HPS-SLS, $\Lambda_{a}=29 \mu \mathrm{m}$ ) have XT of $-17 \mathrm{~dB} / 100 \mathrm{~km}$ applicable for transmission distances up to $60 \mathrm{~km}$ and $15 \mathrm{~km}$ with QPSK and 16 QAM modulation formats, and may be used for ultra-high-density interconnects in the data centers. 

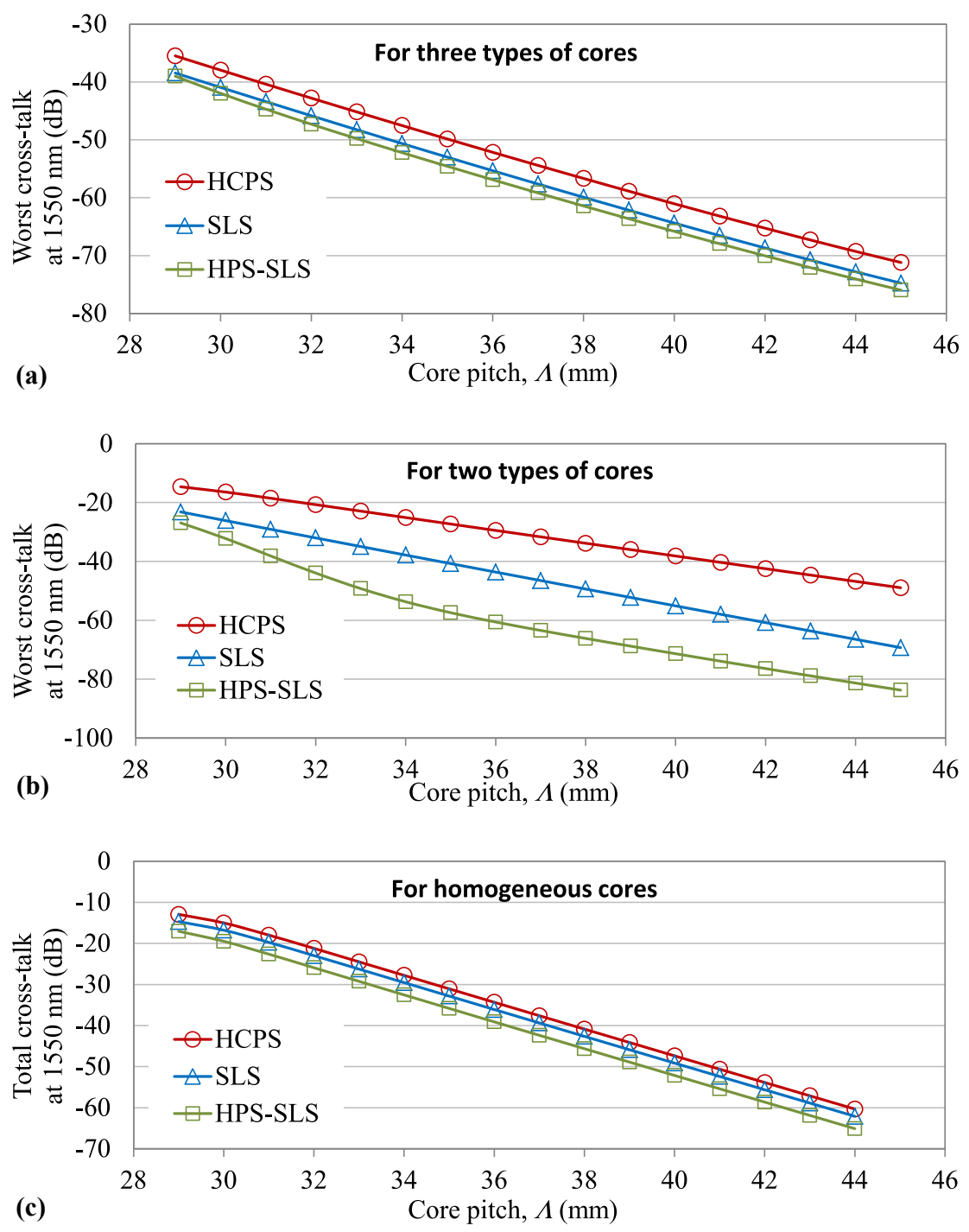

Fig. 6. Average total XT per $100 \mathrm{~km}$ of NC-MCFs with HCPS, SLS, and HPS-SLS at $1550 \mathrm{~nm}$ for (a) heterogeneous core arrangement with three types of cores, (b) heterogeneous core arrangement with two types of cores, and (c) homogeneous core arrangement.

\section{Examples of NC-MCFs}

In this section, we present three example of potential NC-MCFs structures. In all the examples, three types of cores are used to reduce the inter-core XT where each core is surrounded by two other types of cores for long-haul applications. The outer cladding thickness is assumed to be $37 \mu \mathrm{m}$ [7].

\subsection{Elliptical MCFs}

Fig. 7 illustrates examples of SM elliptical MCFs with $b=248 \mu \mathrm{m}$, and $\mathrm{e}=1,2$ and 2.5 in three colors where $\mathrm{e}=1$ corresponds to a circular cross section. In Fig. 7(a), a HPS-SLS structure is shown where there are two core pitches, $\Lambda_{a}=29 \mu \mathrm{m}$ and $\Lambda_{b}=\Lambda_{a} \sqrt{5} / 2=32.4 \mu \mathrm{m}$ as explained in Fig. 2(c). In this case, the number of cores which can be housed with a cladding thickness of $37 \mu \mathrm{m}$ is $33,73,93$ for $\mathrm{e}=1,2,2.5$, respectively. 


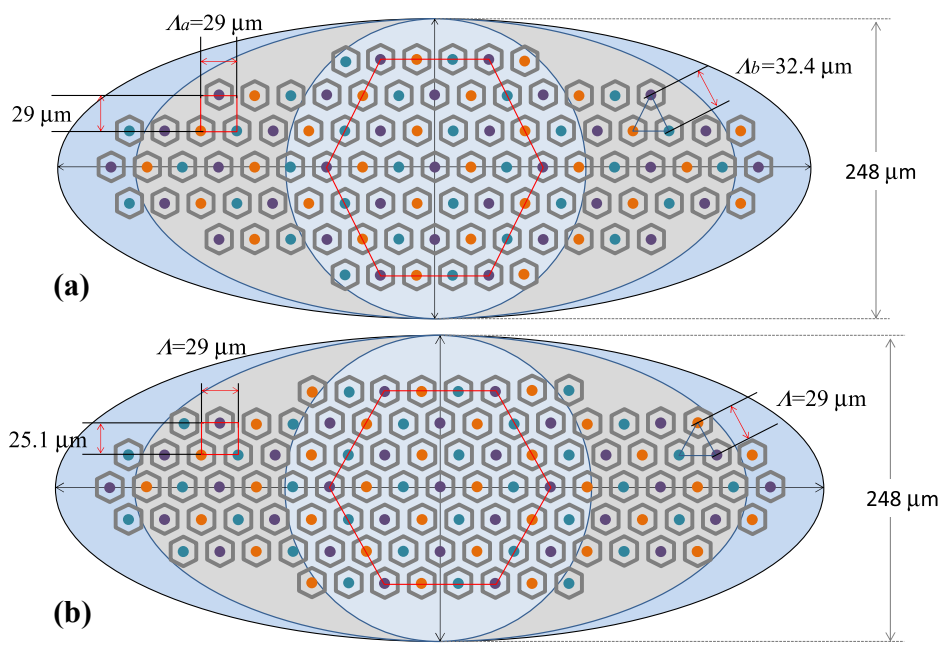

Fig. 7. Various cross-sections of elliptical SM-NC-MCFs for (a) HPSSLS and (b) HCPS both with $\mathrm{b}=248 \mu \mathrm{m}$.

In Fig. 7(b), a HCPS structure is shown where the vertical distance between two rows of cores is $3.88 \mu \mathrm{m}$ less than that of HPS-SLS. Therefore, this structure can accommodate more number of cores compared to HPS-SLS. In this case, the number of cores which can be housed with a cladding thickness of $37 \mu \mathrm{m}$ is 37,81 , 101 for $\mathrm{e}=1,2,2.5$, respectively.

\subsection{Racetrack oval shaped MCFs}

Fig. 8 illustrates examples of racetrack oval cross sectional MCFs with $\mathrm{e}=1,2$ and 2.5. The radius of both circular edges is assumed to be equal to half the cladding diameter in the minor axis b, i.e., $\mathrm{b} / 2$. In this cross section, more number of cores can be accommodated in a given dimension (i.e., $\mathrm{a} \times \mathrm{b}$ ) compared to the aforementioned elliptical MCFs because part of the outer cladding of this structure is straight which reduces the unused spaces in the cladding. In Fig. 8(a), racetrack oval shaped MCFs with HPS-SLS and $b=248 \mu \mathrm{m}$ is shown where there are two

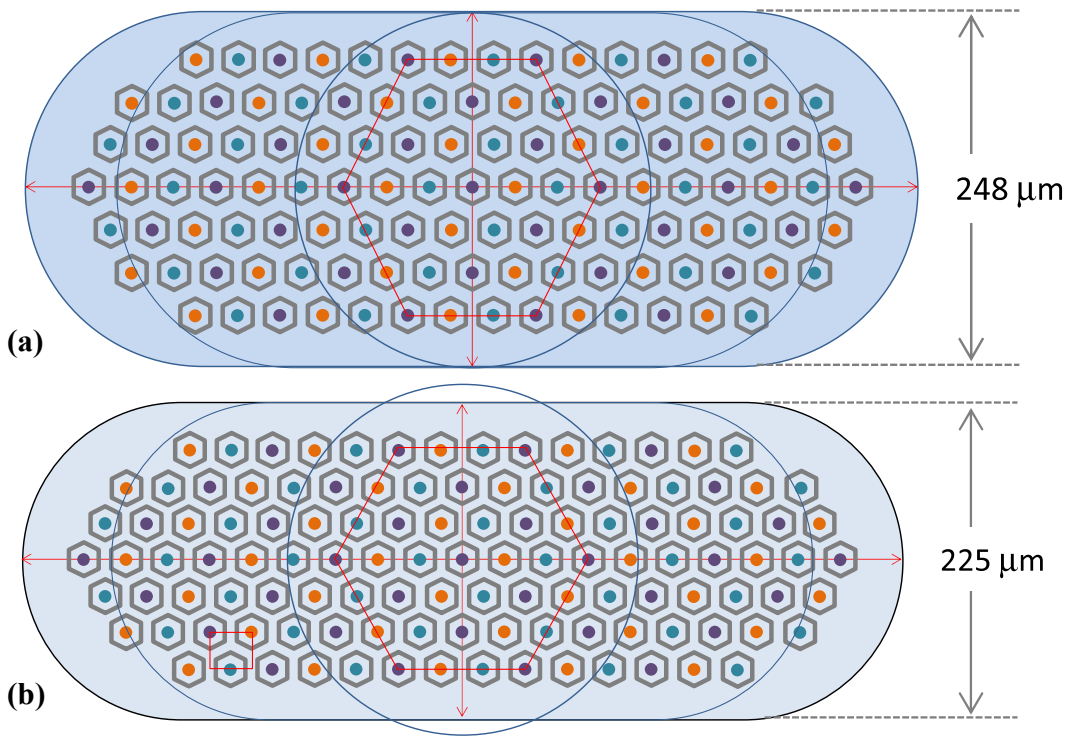


core pitches, $\Lambda_{a}=29 \mu \mathrm{m}$ and $\Lambda_{b}=\Lambda_{a} \sqrt{5} / 2=32.4 \mu \mathrm{m}$ as explained above. The number of cores which can be housed with a cladding thickness of $37 \mu \mathrm{m}$ is 89,119 for $\mathrm{e}=2,2.5$, respectively.

In Fig. 8(b), racetrack oval shaped MCFs with HCPS and $b=225 \mu \mathrm{m}$ is shown. This core arrangement can accommodate nearly the same number of cores within a reduced cross-sectional area compared to the HPS-SLS ones because of the reduced distance between two neighboring horizontal rows. The number of cores which can be housed with a cladding thickness of $37 \mu \mathrm{m}$ is 89,119 for $\mathrm{e}=2,2.5$, respectively, which is same as Fig. 8(a). Reduction in cladding diameter results in an increase in bending flexibility. For example, for 7 rows of cores, a total of $3.88 \times 6=23 \mu \mathrm{m}$ can be reduced in cladding diameter while the core count remains almost the same. This reduction is depicted in Fig. 8(b) by keeping the circle of Fig. 8(a) in its original size.

\subsection{Sharp-cornered oblong shaped MCFs}

Fig. 9 shows examples of sharp-cornered oblong shaped MCFs with $\mathrm{e}=1,1.5,2$ and 2.5. The radius of both circular edges is assumed to be equal to half the cladding diameter in the major axis a, i.e., a/2. The shape can be formed by grinding two sides of a circular preform. Figs. 9(a) and (b) show HPS-SLS and HCPS core arrangements, respectively. Again, HCPS can accommodate almost the same number of cores within a reduced cross-sectional area compared to HPS-SLS. The number of cores which can be housed with a cladding thickness of $37 \mu \mathrm{m}$ is 65, 99, 129 for $\mathrm{e}=1.5,2,2.5$, respectively for both Fig. 9(a) and (b).

(a)
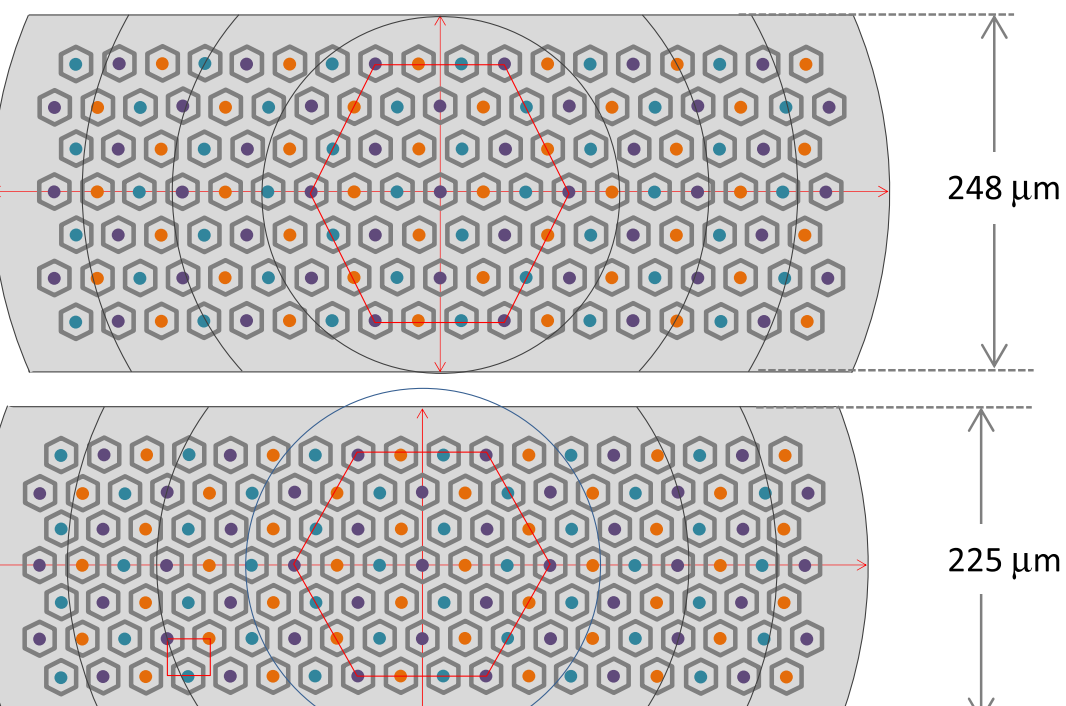

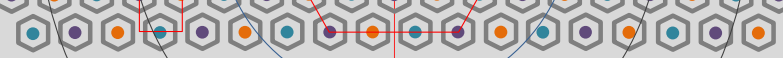

(b)

Fig. 9. Various cross-sections of sharp-cornered oblong shaped SMNC-MCFs for (a) HPS-SLS with $b=248 \mu \mathrm{m}$ and (b) HCPS with $\mathrm{b}=225 \mu \mathrm{m}$.

\subsection{Maximum number of cores in different NC-MCFs}

Fig. 10 summarizes maximum core counts in three types of NC-MCFs proposed for SLS and HCPS core arrangements as the core counts for SLS and HPS-SLS are almost the same. It is obvious that those for elliptical MCFs is the lowest while those for sharp-cornered oblong MCFs the highest. It can be seen that around 100 
cores can be housed in NC-MCFs depending on different parameters. For example, as shown in Fig. 10(c) when $\mathrm{b}$ is $248 \mu \mathrm{m}$ with $\mathrm{e}=2.5(\mathrm{a}=620 \mu \mathrm{m})$, the maximum number of cores in elliptical, racetrack oval shaped and sharp-cornered oblong shaped NC-MCFs with HCPS are 101, 119 and 129, respectively.
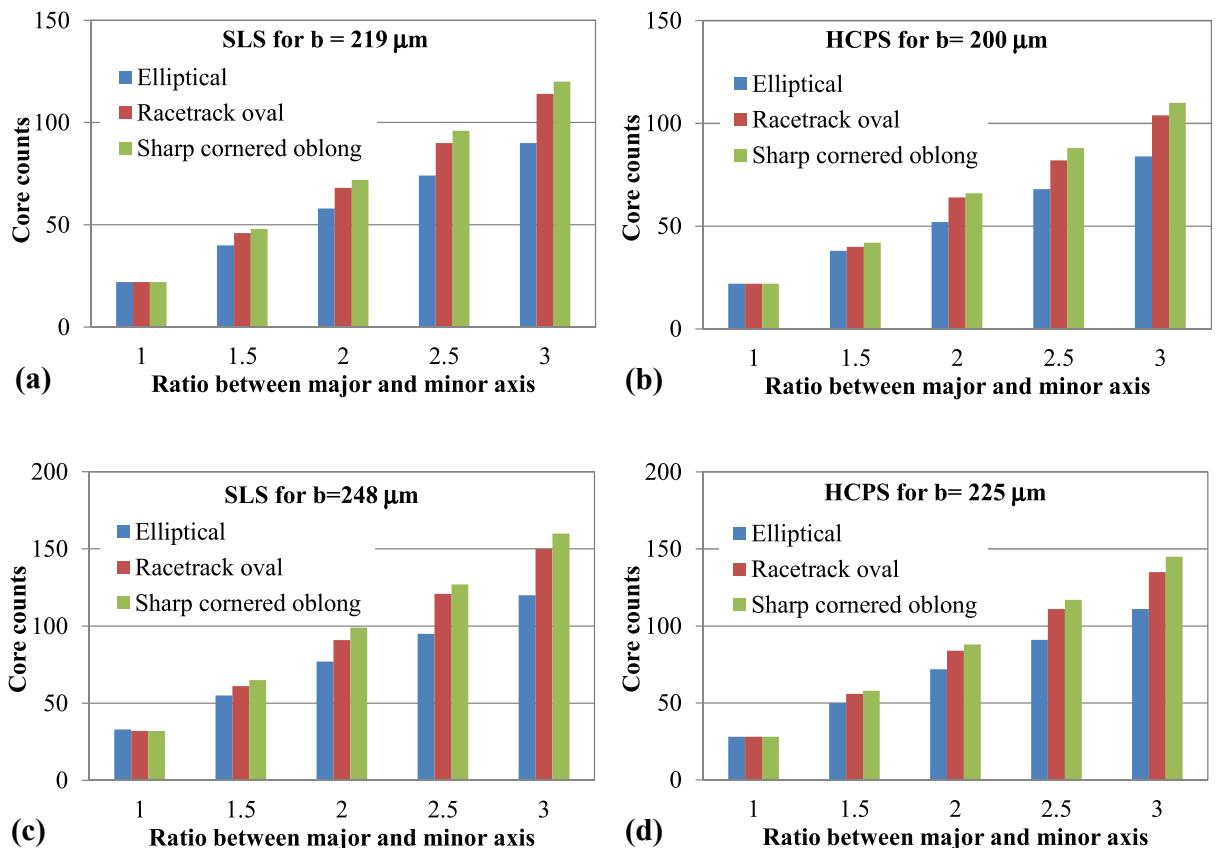

Fig. 10. Comparison of maximum core counts among three NC-MCFs with the same a and $b$.

\section{Mechanical reliability of NC-MCFs}

In this section, we study the mechanical reliability of NC-MCFs. For circular MCFs, the stress on the fiber is considered to be independent of the fiber bending direction while the stress on NC-MCFs highly depends on its direction; the stress will be lower if the fiber is bent in the minor axis while the stress will be higher if the fiber bend in the major axis.
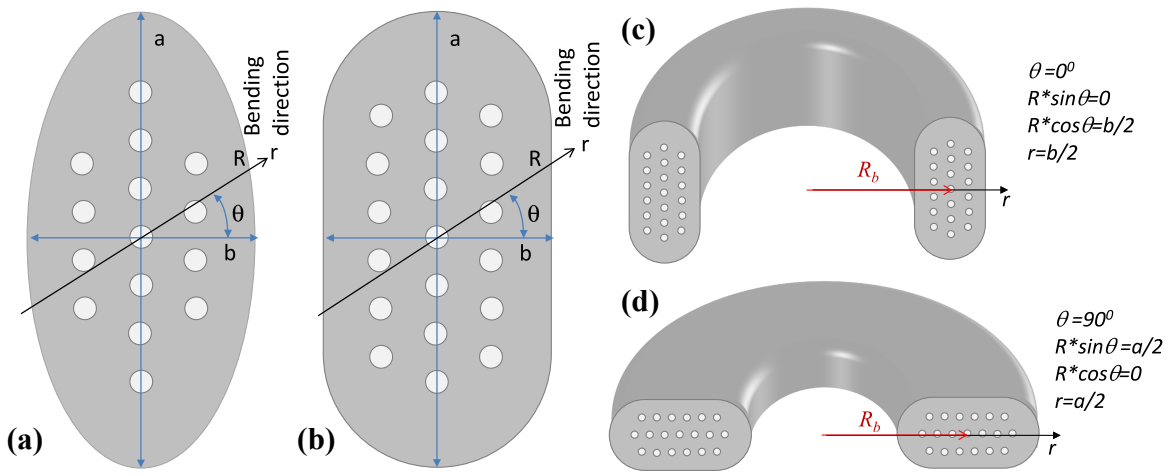

Fig. 11. Bending direction (a) in an elliptical NC-MCF, (b) in a racetrack oval shaped NC-MCF. Fibers bend (c) in the minor axis, and $(\mathrm{d})$ in the major axis.

We investigate the mechanical reliability of NC-MCFs to identify their allow- 


$$
F=1-\exp \left(N_{p} L\left\{1-\left[1+\left(\frac{\sigma}{\sigma_{p}}\right)^{n} \frac{t}{t_{p}}\right]^{\frac{m}{n-2}}\right\}\right)
$$

where $N_{p}$ is the average number of failures per unit length during proofing, $L$ is the effective fiber length, $m$ is the Weibull distribution parameter, $n$ is the crack growth parameter (stress corrosion parameter), $t_{p}$ is the proof time, $t$ is the life time of the fiber, $\sigma_{p}$ is the proof level, and $\sigma$ is the maximum bending stress. In the calculation, we set $N_{p}, n, t_{p}, t, \sigma_{p}$ to be $10^{-4} / \mathrm{m}, 20,1 \mathrm{sec}$., 20 years and $1 \%$ or $2 \%$.

We assume the fiber bend in the direction indicated by $r$ as shown in Fig. 11(a) and (b). The bending radius is $R_{b}, \mathrm{e}=\mathrm{a} / \mathrm{b}$ is the ratio of the cladding diameter in the major axis to that in the minor axis, $E$ is the Young's modulus, and $\theta$ is the angle between the minor axis and the direction of bending. The axial stress $\sigma$ is approximated by the following equation $[16,17]$

$$
\sigma(r)=\frac{E}{R_{b}} r .
$$

Figs. 12 and 13 show the calculated failure probability as a function of crosssectional area of elliptical MCFs with bending radii of $30 \mathrm{~mm}$ and $60 \mathrm{~mm}$, respectively for the conditions of (a) $1 \%$ proof level, e $=2$, (b) $1 \%$ proof level, $\mathrm{e}=2.5$, (c) $2 \%$ proof level, $\mathrm{e}=2$, and (d) $2 \%$ proof level, $\mathrm{e}=2.5$. The black solid curves in Figs. 12 and 13 correspond to circular MCFs with bending radii of $30 \mathrm{~mm}$ and $60 \mathrm{~mm}$, respectively and the horizontal dotted line indicates a guideline failure probability of $3.2 \times 10^{-6}$ when standard single mode fibers with a $125 \mu \mathrm{m}$ cladding diameter are bent. It can be seen that if the bending angle $\theta$ is within $30^{\circ}$, elliptical MCFs can have larger cross sectional areas than circular MCFs, enabling higher core counts. Two shaded areas in the figures $\left(79.5 \times 10^{-9} \mathrm{~m}^{2}-96.6 \times 10^{-9} \mathrm{~m}^{2}\right.$ and $99.4 \times 10^{-9} \mathrm{~m}^{2}-120.8 \times 10^{-9} \mathrm{~m}^{2}$ ) correspond to cross-sectional areas which can house around $70-85$ cores and $90-105$ cores in the case of SM-MCFs. It is seen that in order to accommodate more than 70 cores, $\theta \approx 0$ degree (bent in the direction of minor axis) should be necessary for $2 \%$ proof level, $R_{b}=30 \mathrm{~mm}$ and $1 \%$ proof level, $R_{b}=60 \mathrm{~mm}$ while for $2 \%$ proof level, $R_{b}=60 \mathrm{~mm}, \theta$ is relaxed to $\leq 30^{\circ}$.
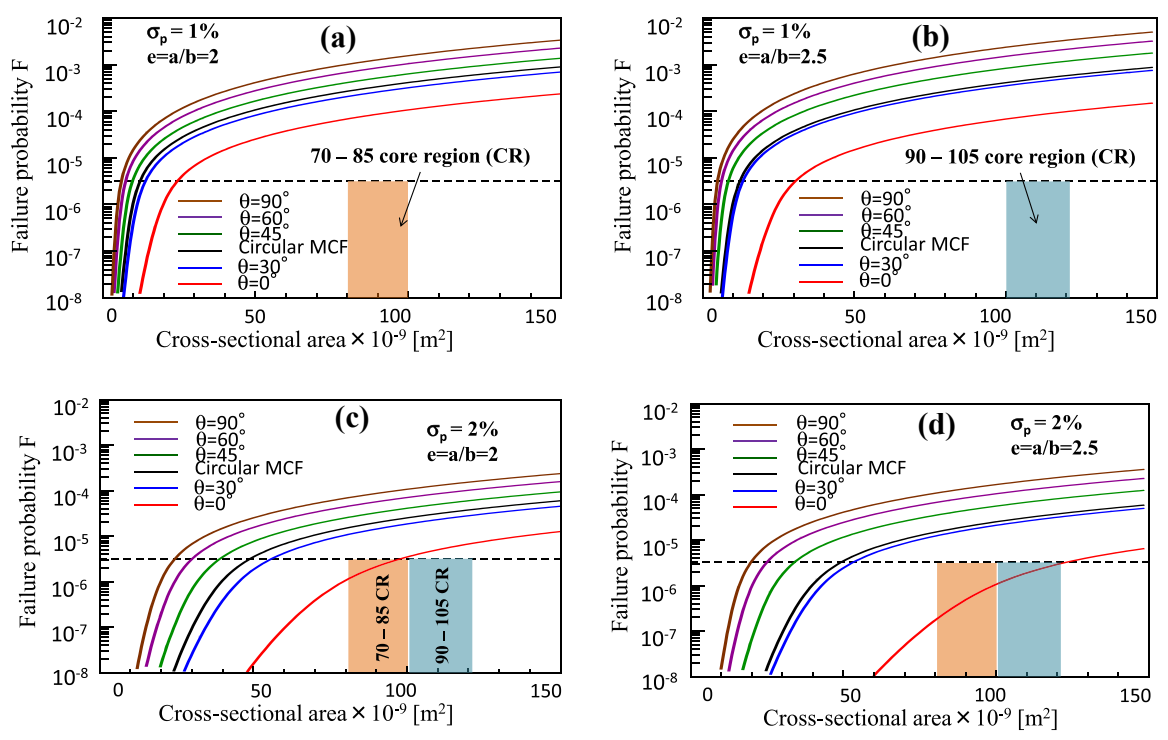

Fig. 12. Failure probability for elliptical MCFs with $R_{b}=30 \mathrm{~mm}$. 

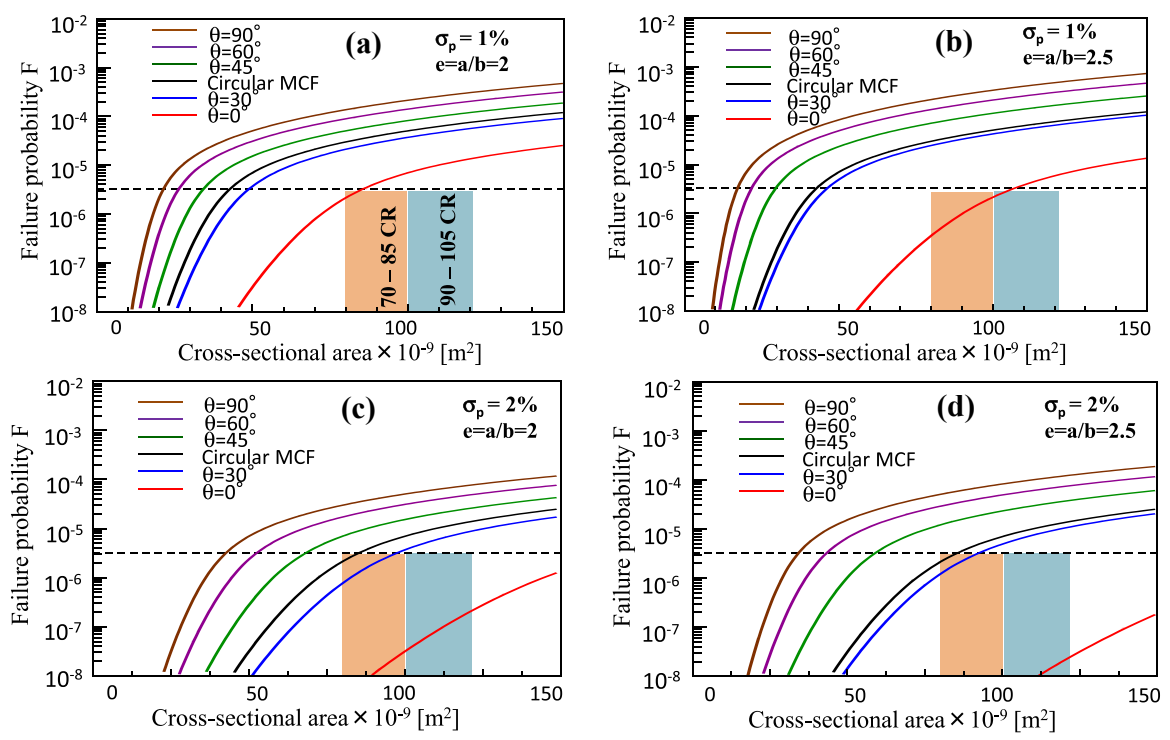

Fig. 13. Failure probability for elliptical MCFs with $R_{b}=60 \mathrm{~mm}$.

Figs. 14 and 15 show the calculated failure probability as a function of crosssectional area of racetrack-oval shaped MCFs with $30 \mathrm{~mm}$ and $60 \mathrm{~mm}$ bending radii, respectively for the conditions of (a) $1 \%$ proof level, e $=2$, (b) $1 \%$ proof level, e $=2.5$, (c) $2 \%$ proof level, e $=2$, and (d) $2 \%$ proof level, e $=2.5$. As in Figs. 12 and 13, the black solid curves in Figs. 14 and 15 correspond to circular MCFs with bending radii of $30 \mathrm{~mm}$ and $60 \mathrm{~mm}$, respectively and the horizontal dotted line indicates a guideline failure probability of $3.2 \times 10^{-6}$.
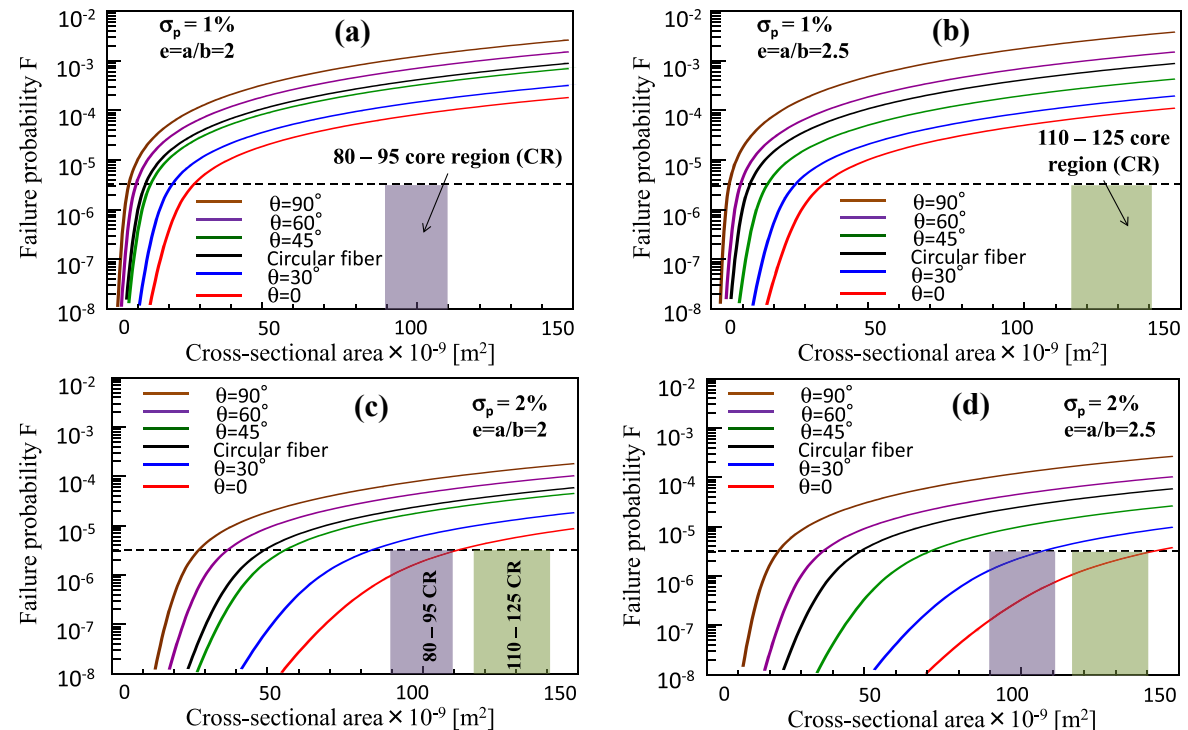

Fig. 14. Failure probability for racetrack oval shaped MCFs with $R_{b}=30 \mathrm{~mm}$.

It can be seen that if the bending angle $\theta$ is within $45^{\circ}$, racetrack-oval shaped MCFs can have larger cross sectional areas than circular MCFs, enabling higher core counts. Two shaded areas in the figures $\left(90.4 \times 10^{-9} \mathrm{~m}^{2}-109.8 \times 10^{-9} \mathrm{~m}^{2}\right.$ and $115.7 \times 10^{-9} \mathrm{~m}^{2}$ to $140.6 \times 10^{-9} \mathrm{~m}^{2}$ ) correspond to cross-sectional areas which can house around $80-95$ cores and 110-125 cores in the case of SM-MCFs. It is seen that in order to accommodate more than 80 cores, $\theta \leq 30^{\circ}$ should be necessary for $2 \%$ proof level, $R_{b}=30 \mathrm{~mm}, \mathrm{e}=2.5$ and $1 \%$ proof level, $R_{b}=60 \mathrm{~mm}, \mathrm{e}=2.5$ while for $2 \%$ proof level, $R_{b}=60 \mathrm{~mm}, \theta$ is relaxed to $\leq 45^{\circ}$ as shown in Figs. 14 
and 15 . It should be noted that for $2 \%$ proof level, $R_{b}=60 \mathrm{~mm}$, e $\geq 2$, more than 100 cores can be accommodated with $\theta \leq 30^{\circ}$, and for $2 \%$ proof level, $R_{b}=60 \mathrm{~mm}, \mathrm{e}=2.5, \theta$ is relaxed to $\leq 45^{\circ}$ as shown in Fig. 15(d).

For sharp-cornered oblong shaped SM-NC-MCFs, it is expected that they have lower failure probabilities for the same cross sectional area than racetrack-oval shaped MCFs do (Figs. 14 and 15) since sharp-cornered oblong shaped SM-NCMCFs have larger cross sectional areas than racetrack-oval shaped ones for the same $\mathrm{e}=\mathrm{a} / \mathrm{b}$.
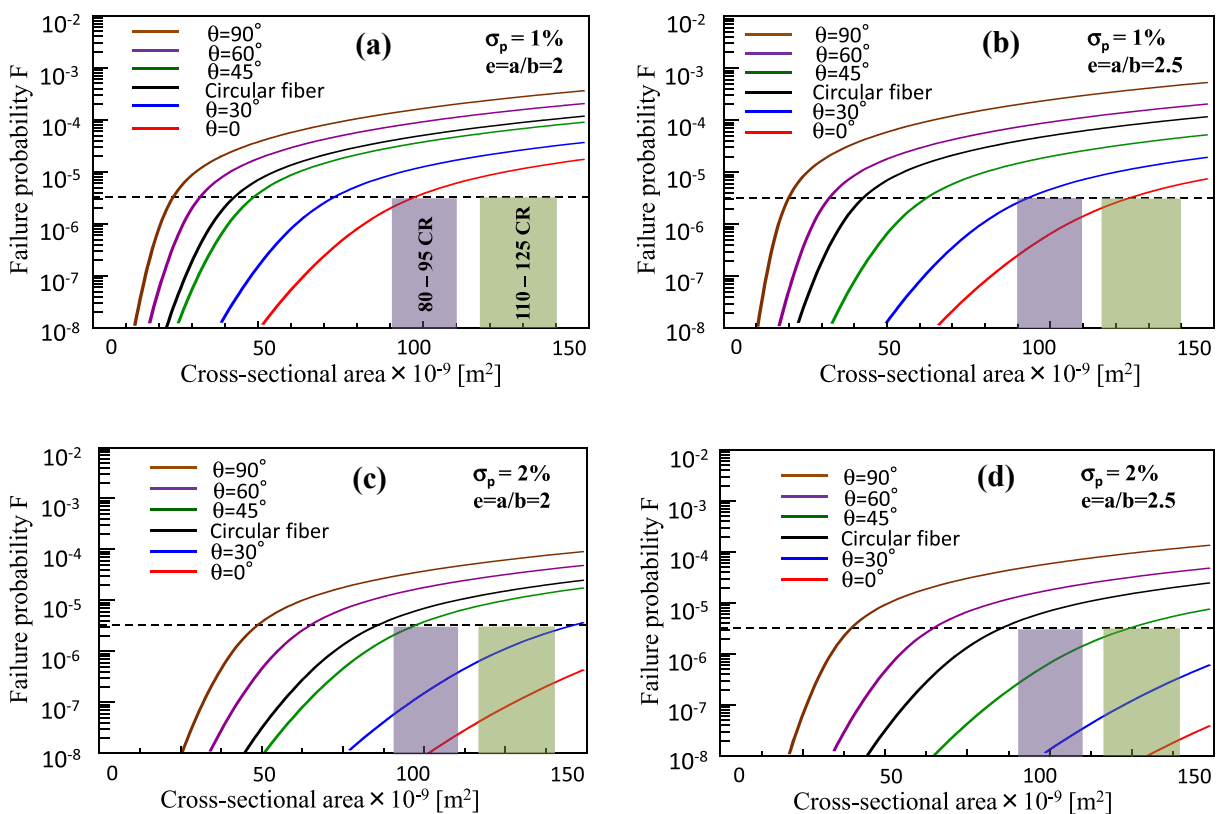

Fig. 15. Failure probability for racetrack oval shaped MCFs with $R_{b}=60 \mathrm{~mm}$.

We assume the fiber will in general bend in the direction of minor axis [12] although in practice, $\theta$ might not be zero. Based on the results above, it is obvious that elliptical cross-sectional MCFs may not be a good choice for achieving ultra large core count fiber as it limits the angle $\theta$ most strictly. On the other hand, racetrack oval shapes will be promising as it allows $\theta$ up to 45 degrees, and we assume there will be many potential applications where it will be possible to maintain this bending restrictions.

\section{Conclusion}

We propose NC-MCFs with elliptical, racetrack oval shaped, and sharp-cornered oblong shaped cladding cross sections for super-dense space-division multiplexing. The new MCFs are expected to accommodate more than 100 cores per fiber while keeping the machanical reliability comparable to that of SMFs.

\section{Acknowledgments}

This work was supported by the EU-Japan coordinated R\&D project on "Scalable and Flexible Optical Architecture for Reconfigurable Infrastructure (SAFARI)" commissioned by the Ministry of Internal Affairs and Communications of Japan and EC Horizon 2020, Innovationsfonden e-space (0603-00514B) and SPOC research centre of excellence, ref DNRF123. 